\title{
A VIOLÊNCIA NO AMBIENTE UNIVERSITÁRIO
}

\section{Violence in the university environment}

\section{La violencia en el ambiente de la universidad}

\author{
Carla Christina Pereira da Silva Godinho \\ Universidade de Fortaleza - UNIFOR - Fortaleza (CE) - Brasil \\ Faculdade Ateneu - FATE - Fortaleza (CE) - Brasil
}

Soraya da Silva Trajano

Universidade de Fortaleza - UNIFOR - Fortaleza (CE) - Brasil

Cleibiane Vituriano de Souza

Universidade de Fortaleza - UNIFOR - Fortaleza (CE) - Brasil

\section{Natasha Teixeira Medeiros}

Universidade de Fortaleza - UNIFOR - Fortaleza (CE) - Brasil

Universidade Federal do Piauí - UFPI - Parnaíba (PI) - Brasil

\section{Ana Maria Fontenelle Catrib}

Universidade de Fortaleza - UNIFOR - Fortaleza (CE) - Brasil

Ana Paula Vasconcellos Abdon

Universidade de Fortaleza - UNIFOR - Fortaleza (CE) - Brasil

\section{RESUMO}

Objetivo: Avaliar a visão do estudante sobre a situação de violência no ambiente universitário e os possíveis fatores associados. Métodos: Estudo transversal e analítico em uma instituição de ensino superior privada do município de Fortaleza, Ceará, realizado entre abril a novembro de 2017. Participaram 512 estudantes dos cursos do Centro de Ciências da Saúde. Utilizouse o Instrumento de Avaliação da Promoção da Saúde na Universidade (IAPSU) para coletar as variáveis sociodemográficas, acadêmicas e a situação de violência. As associações foram testadas pelo Teste do qui-quadrado, por meio do programa SPSS versão 20.0 Resultados: Os estudantes dos cursos da área da saúde apresentaram-se com idade $\leq 24$ anos ( $n=393 ; 76,8 \%)$, sexo feminino $(n=357 ; 69,7 \%)$, raça branca $(n=261 ; 51,0 \%)$ e católicos $(n=332 ; 64,8 \%)$. Uma proporção de $68,4 \%(n=350)$ participa em atividades promovidas pela universidade e $56,4 \%(n=289)$ em atividades extracurriculares. A situação de violência no ambiente universitário foi apontada por 30,9\% ( $n=158)$, sendo do tipo psicológica $(n=132 ; 84,1 \%)$ e praticada pelos próprios estudantes $(n=115 ; 72,8 \%)$. Na análise multivariada foi verificada associação significativa da situação de violência na universidade com o último ano $(O R=2,315 ; p=0,004)$ e com o tratamento entre os universitários $(O R=0,476 ; p=0,048)$. Conclusão: A situação de violência do tipo psicológica está presente no ambiente universitário estudado, é perpetrada pelos próprios estudantes, estando associada ao último ano do curso de graduação na área da saúde e ao tratamento entre os estudantes.

Descritores: Violência; Educação Superior; Universidade.

\section{ABSTRACT}

Objective: To evaluate the student's opinion about violence in the university environment and potential associated factors. Methods: This analytical cross-sectional study was carried out at a private higher education institution in the municipality of Fortaleza, Ceará, between April and November 2017. Participants were 512 students enrolled in schools of the Health Sciences Center. The Assessment Tool for Health Promotion at the University (Instrumento de Avaliação da Promoção da Saúde na Universidade - IAPSU) was used to collect sociodemographic, academic and violence data. Associations were tested by the chi-squared test in SPSS version 20.0. Results: Students enrolled in health schools were aged $\leq 24$ years ( $n=393 ; 76.8 \%)$, women ( $n=357$; $69.7 \%)$, White $(n=261 ; 51.0 \%)$ and Catholic $(n=332 ; 64.8 \%)$. A total of $68.4 \%(n=350)$ of the students participated in activities promoted by the university and $56.4 \%(n=289)$ participated in extracurricular activities. Violence in the university environment was reported by $30.9 \%(n=158)$ of the participants, particularly psychological violence $(n=132 ; 84.1 \%)$ and violence perpetrated by the

Este artigo foi selecionado, corrigido e aprovado para publicação pelo Vı Seminário Internacional em Promoção da Saúde, seguindo suas normas e formatação. 
students themselves ( $n=115 ; 72.8 \%)$. In the multivariate analysis, violence in the university was significantly associated with the last year $(O R=2,315 ; p=0.004)$ and relationship between university students $(O R=0.476 ; p=0.048)$. Conclusion: Psychological violence is present in the university environment and is perpetrated by the students themselves. It is associated with the last year of undergraduate health school and relationship between students.

Descriptors: Violence; Education, Higher; University.

\section{RESUMEN}

Objetivo: Evaluar la opinión del estudiante sobre la situación de violencia en el ambiente universitario y los posibles factores asociados. Métodos: Estudio transversal y analítico realizado en una institución de educación superior privada del municipio de Fortaleza, Ceará entre abril y noviembre de 2017. Participaron 512 estudiantes de los cursos del Centro de Ciencias de la Salud. Se utilizó el Instrumento de Evaluación de la Promoción de la Salud en la Universidad (IEPSU) para recoger datos de las variables sociodemográficas, académicas y de situación de violencia. Se aplicó la prueba Chi-cuadrado para las asociaciones a través del programa SPSS versión 20.0 Resultados: Los estudiantes de los cursos del área de la salud tenían edad $\leq 24$ años ( $n=393 ; 76,8 \%)$, eran del sexo femenino ( $n=357 ; 69,7 \%)$, de la raza blanca $(n=261 ; 51,0 \%)$ y católicos $(n=332 ; 64,8 \%)$. Una proporción del 68,4\% ( $n=350)$ participa de actividades promovidas por la universidad y el 56,4\% ( $n=289)$ de actividades extracurriculares. La situación de violencia en el ambiente de la universidad ha sido apuntada por el 30,9\% (n=158) como tipo psicológica ( $n=132 ; 84,1 \%)$ y practicada por los propios estudiantes $(n=115 ; 72,8 \%)$. En el análisis multivariado ha sido identificada una asociación significativa de situación de violencia en la universidad con el último año $(O R=2,315 ; p=0,004)$ y con el trato entre los universitarios $(O R=0,476 ; p=0,048)$. Conclusión: La situación de violencia del tipo psicológica está presente en el ambiente universitario estudiado, es realizada por los propios estudiantes y está asociada con el último año del curso de graduación del área de la salud y al trato entre los estudiantes.

Descriptores: Violencia; Educación Superior; Universidades.

\section{INTRODUÇÃO}

A violência é um problema social e ocorre quando há algum tipo de conduta hostil causada por um agressor contra uma vítima. É uma relação de dominação, em que a vítima não consegue defender-se e com isso é exposta a abusos diversos ${ }^{(1)}$.

Em face ao atual panorama brasileiro, destacadamente permeado pelo aumento gradativo da violência e seu efeito destrutivo, as instituições de ensino têm oferecido maior atenção a essas questões. As universidades buscaram desenvolver pesquisas sobre a violência, considerando que é um fenômeno social, complexo, multicausal e histórico que atinge toda a sociedade em diferentes graus. As reflexões acerca desta temática, parecem favorecer com o fomento de soluções e estratégias no sentido de minimizar o fenômeno da violência, desde que haja o envolvimento de diferentes setores e ações na operacionalização das estratégias de prevenção e intervenções ${ }^{(2)}$.

Independente da tipologia, a violência traz impactos negativos tanto na saúde física como mental, sendo fundamental o diagnóstico, análise e propositura de estratégias inclusive sob o ponto de vista de saúde pública, na perspectiva de discuti-la no ambiente universitário( ${ }^{(3)}$, notadamente nos cursos de graduação na área da saúde, prevenindo a banalização do abuso, assédio e estelionato(4) e assim, contribuindo por legitimar o papel promotor da saúde que tem a universidade.

O trote, o bullying e a homofobia são exemplos de situações de violência nas instituições de ensino superior (IES), que geram implicações nas relações interpessoais, ruptura de comportamentos éticos ${ }^{(5)}$, danos psíquicos e comprometimento da qualidade de vida ${ }^{(6,7)}$.

Nesse dinâmico e multifacetado contexto de saúde nos espaços educacionais, ratifica-se a importância da realização um diagnóstico situacional de quais fatores estão diretamente relacionados com as situações de violência no ambiente universitário, cujas informações podem favorecer reflexões entre os sujeitos educacionais e IES, e fomentar direcionamentos, pactuações e estratégias preventivas perenes de não violência no campus.

Diante disso, este estudo objetivou avaliar a visão do estudante sobre a situação de violência no ambiente universitário e os possíveis fatores associados.

\section{MÉTODOS}

Estudo transversal e analítico, desenvolvido na Universidade de Fortaleza (UNIFOR), Ceará, proveniente de um projeto guarda-chuva intitulado "Promoção da saúde na população jovem: qual papel da universidade?", no período de abril a novembro de 2017. 
A população de estudo foi composta por estudantes ( $\geq 18$ anos), independente do sexo e regularmente matriculados, em um dos nove cursos da área da saúde, selecionados para pesquisa. Excluíram-se deficientes visuais, físicos e gestantes devido às particularidades dos instrumentos não previstos no projeto guarda-chuva.

Estimou-se um quantitativo pelo cálculo amostral considerando uma população finita (7.000 estudantes da área da saúde na instituição), prevalência de $50.0 \%$ (adotada quando há desconhecimento), intervalo de confiança de $95 \%$, erro máximo de $5 \%$, taxa de não resposta de $20 \%$. O quantitativo amostral foi inicialmente calculado em 417 estudantes.

No entanto, para propiciar distribuição equitativa entre os cursos e semestres participaram 544 estudantes, sendo retirados 32 devido à inconsistência ou erros no preenchimento do instrumento de coleta. Assim, permaneceram 512 distribuídos simetricamente por cursos, sendo 57 do curso de Educação Física, 57 de Enfermagem, 58 de Farmácia, 58 de Fisioterapia, 46 de Fonoaudiologia, 58 de Medicina, 60 de Nutrição, 59 de Odontologia e 59 de Psicologia.

Para a seleção da amostra, estratificada por curso de graduação, foi realizado levantamento das disciplinas dos semestres específicos (primeiro e último ano), em diferentes turnos e horários, e os universitários destas disciplinas foram convidados a participar.

Para coleta, utilizou-se o Instrumento de Avaliação da Promoção da Saúde na Universidade - IAPSU, autoaplicável e validado ${ }^{(8)}$. Apresenta 41 itens organizados em duas partes, a primeira para coletar características sociodemográficas; e a segunda possui cinco domínios: 1) atividade física, 2) alimentação, 3) fatores ambientais, 4) fatores psicossociais e consumo de álcool e drogas e 5) Práticas Integrativas e Complementares. Neste estudo, analisaram-se variáveis sociodemográficas (idade, sexo, raça e religião), formação acadêmicas (curso de graduação e semestre letivo) e variáveis do domínio 4 (participação em atividades promovidas pela universidade e extracurriculares, situações de violência, segurança e tratamento de grupos vulneráveis).

Aplicou-se estatística descritiva e inferencial pelo programa IBM ${ }^{\circledR}$ SPSS Statistics versão 20.0, sendo as variáveis qualitativas descritas por meio da frequência relativa (\%) e absoluta (n). Para análise inferencial algumas variáveis foram dicotomizadas: idade categorizada ( $\leq 24$ anos e $\geq 25$ anos), raça categorizada (branca e não branca), religião (católica, não católica e sem religião), atividades promovidas pela universidade e extracurriculares ("sim" para participação em no mínimo uma ou "não") e segurança na universidade ("sim" quando seguro ou muito seguro e "não" quando razoavelmente, pouco ou nada seguro).

$\mathrm{Na}$ análise bivariada, verificou-se associação entre a variável dependente (situação de violência no ambiente universitário) e as independentes (sociodemográficas, acadêmicas, participação em atividades e segurança) pela aplicação do teste qui-quadrado, seguido do cálculo Odds Ratio (OR) bruto e seus respectivos intervalos de confiança (IC), com nível de significância de $5 \%$.

Posteriormente, realizou-se a análise de regressão multivariada, considerando $p<0,20$, a fim de ajustar possíveis efeitos de confusão. Para construção do modelo final, utilizou-se a seleção hierarquizada de fatores, e estimou-se o OR ajustado.

Este estudo foi aprovado pelo comitê de ética da Universidade de Fortaleza (Parecer $n^{\circ}$. 1.795.390). Todos os participantes assinaram o Termo de Consentimento Livre e Esclarecido.

\section{RESULTADOS}

O perfil sociodemográfico apresentou maior proporção de estudantes na faixa etária igual ou inferior a 24 anos ( $n=393 ; 76,8 \%)$, sexo feminino $(n=357 ; 69,7 \%)$, raça branca $(n=261 ; 51,0 \%)$ e da religião católica $(n=332 ; 64,8 \%)$ (Tabela I).

A participação em pelo menos uma atividade promovida pela universidade foi manifestada por 350 (68,4\%) estudantes, destacadamente $232(45,3 \%)$ em participação social e $231(45,1 \%)$ em comportamentos promotores de saúde. Com relação à participação em atividades extracurriculares, $289(56,4 \%)$ participavam de pelo menos uma, sendo 173 (33,8\%) monitoria e $116(22,7 \%)$ em centro acadêmico (Tabela I).

Ao analisar a situação de violência, segurança e tratamento de grupos vulneráveis no ambiente universitário, $158(30,9 \%)$ informaram situação de violência na universidade, principalmente do tipo psicológica $(n=132 ; 84,1 \%)$ e cujos praticantes foram os próprios alunos $(n=115 ; 72,8 \%)$. O bom tratamento entre os universitários foi referido por $424(82,8 \%)$ e entre universitários e professores por 447 (87,3\%). A sensação de segurança na universidade foi apontada por $331(64,6 \%)$ estudantes. Com relação ao tratamento violento de grupos vulneráveis, foram encontrados os valores correspondentes a $179(35,0 \%)$ em relação aos obesos, $146(28,5 \%)$ aos homoafetivos e $102(20,0 \%)$ em relação aos deficientes físicos (Tabela II). 
Tabela I - Perfil sociodemográfico dos estudantes e participação em atividades promovidas pela universidade e extracurriculares. Fortaleza, Ceará, 2017.

\begin{tabular}{|c|c|c|}
\hline Variáveis & $\mathbf{n}$ & $\%$ \\
\hline \multicolumn{3}{|l|}{ Idade (anos) } \\
\hline$\leq 24$ & 393 & 76,8 \\
\hline$\geq 25$ & 119 & 23,2 \\
\hline \multicolumn{3}{|l|}{ Sexo } \\
\hline Feminino & 357 & 69,7 \\
\hline Masculino & 155 & 30,3 \\
\hline \multicolumn{3}{|l|}{ Raça autorreferida } \\
\hline Branca & 261 & 51,0 \\
\hline Não branca & 251 & 49,0 \\
\hline \multicolumn{3}{|l|}{ Religião ( $n=511)$} \\
\hline Católico & 332 & 65,0 \\
\hline Não católico & 131 & 25,6 \\
\hline Nenhuma & 48 & 9,4 \\
\hline \multicolumn{3}{|l|}{ Atividades promovidas pela universidade } \\
\hline Participação social & 232 & 45,3 \\
\hline Comportamentos promotores de saúde & 231 & 45,1 \\
\hline Recreativas e lazer & 168 & 32,8 \\
\hline Cidadania & 159 & 31,1 \\
\hline Planejamento de vida & 145 & 28,3 \\
\hline Convivência universitária & 144 & 28,1 \\
\hline Afetividade e relacionamentos interpessoais & 142 & 27,7 \\
\hline Culturais & 122 & 23,8 \\
\hline Autoestima e autoconhecimento & 121 & 23,6 \\
\hline Reflexões sobre sexualidade & 103 & 20,1 \\
\hline \multicolumn{3}{|l|}{ Participação em atividade extracurricular } \\
\hline Monitoria & 173 & 33,8 \\
\hline Centro acadêmico & 116 & 22,7 \\
\hline Extensão universitária & 94 & 18,4 \\
\hline Iniciação científica & 88 & 17,2 \\
\hline
\end{tabular}

Tabela II - Análise da situação de violência, segurança e tratamento de grupos vulneráveis no ambiente universitário. Fortaleza, Ceará, 2017.

\begin{tabular}{lcc}
\hline Variáveis & $\mathbf{n}$ & \% \\
\hline Situação de violência na universidade & 158 & 30,9 \\
Tipos de violência & 132 & 84,1 \\
Psicológica & 81 & 51,3 \\
Gênero & 67 & 42,4 \\
Física & 53 & 33,8 \\
$\quad$ Sexual & 115 & 72,8 \\
Praticante de violência & 55 & 35,0 \\
$\quad$ Alunos & 20 & 12,7 \\
$\quad$ Professores & 70 & 44,3 \\
Funcionários & & \\
Pessoas externas à instituição & 424 & 82,8 \\
Tratamento entre os universitários & 88 & 17,2 \\
Bem & & \\
Mal & 447 & 87,3 \\
Tratamento entre universitários e professores & 65 & 12,7 \\
$\quad$ Bem & & 35,0 \\
Mal & 179 & 28,5 \\
Tratamento violento de grupos vulneráveis na universidade & 146 & 20,0 \\
$\quad$ Obeso & 102 & 64,6 \\
Homoafetivo & & 35,4 \\
$\quad$ Deficiente físico & 331 & 181 \\
Sensação de segurança na universidade & & \\
$\quad$ Sim & & \\
Não &
\end{tabular}


A análise bivariada da situação de violência na universidade mostrou associação significativa com os últimos semestres $(O R=2,377 ; p=0,000)$, curso de graduação $(p=0,004)$, principalmente dos cursos de graduação em Fisioterapia $(n=25 ; 15,8 \%)$, Medicina $(n=24 ; 15,2 \%)$ e Psicologia $(n=24 ; 15,2 \%)$, sensação de insegurança na universidade $(O R=2,891 ; p=0,000)$, tratamento entre os universitários $(O R=0,275 ; p=0,000)$ e tratamento entre universitários e professores (OR: 0,376, p=0,000) (Tabela III).

$\mathrm{Na}$ análise multivariada foi verificada associação significativa da situação de violência na universidade somente com o último ano ( $O R=2,315 ; p=0,004)$ e com o tratamento entre os universitários $(O R=0,476 ; p=0,048)$ (Tabela IV).

Tabela III - Análise bivariada da situação de violência na universidade com as variáveis sociodemográficas e acadêmicas. Fortaleza, Ceará, 2017.

\begin{tabular}{|c|c|c|c|c|}
\hline \multirow{2}{*}{ Variáveis } & \multicolumn{4}{|c|}{ Situação de violência na universidade } \\
\hline & n (\%) & OR bruto & $\mathrm{IC} 95 \%$ & p-valor \\
\hline $\begin{array}{l}\text { Sociodemográficas } \\
\text { Idade }\end{array}$ & & & & 0,772 \\
\hline$\leq 24$ & $120(75,9)$ & 1 & & \\
\hline$\geq 25$ & $38(24,1)$ & 1,067 & $0,687-1,659$ & \\
\hline Sexo & & & & 0,555 \\
\hline Masculino & $45(28,5)$ & 1 & & \\
\hline Feminino & $113(71,5)$ & 1,132 & $0,750-1,710$ & \\
\hline Religião & & & & 0,602 \\
\hline Nenhuma & $16(10,1)$ & 1 & & \\
\hline Não católica & $36(22,8)$ & 0,758 & $0,372-1,545$ & \\
\hline Católica & $106(67,1)$ & 0,938 & $0,493-1,784$ & \\
\hline Raça & & & & 0,498 \\
\hline Não branco & $81(51,3)$ & 1 & & \\
\hline Branco & $77(48,7)$ & 0,878 & $0,604-1,278$ & \\
\hline Acadêmicas & & & & \\
\hline Semestre & & & & $0,000^{*}$ \\
\hline Primeiro ano & $55(34,8)$ & 1 & & \\
\hline Último ano & $103(65,2)$ & 2,377 & $1,611-3,506$ & \\
\hline Graduação & & & & $0,004^{*}$ \\
\hline Educação Física & $11(7,0)$ & - & - & \\
\hline Enfermagem & $13(8,2)$ & - & - & \\
\hline Farmácia & $13(8,2)$ & - & - & \\
\hline Fisioterapia & $25(15,8)$ & - & - & \\
\hline Fonoaudiologia & $17(10,8)$ & - & - & \\
\hline Medicina & $24(15,2)$ & - & - & \\
\hline Nutrição & $11(7,0)$ & - & - & \\
\hline Odontologia & $20(12,7)$ & - & - & \\
\hline Psicologia & $24(15,2)$ & - & - & \\
\hline Participação em atividades & & & & \\
\hline Promovidas pela universidade & & & & 0,304 \\
\hline Não & $45(28,5)$ & 1 & & \\
\hline Sim & $113(71,5)$ & 1,240 & $0,822-1,869$ & \\
\hline Extracurriculares & & & & 0,353 \\
\hline Não & $64(40,5)$ & 1 & & \\
\hline $\operatorname{Sim}$ & $94(59,5)$ & 1,198 & $0,819-1,752$ & \\
\hline Sensação de segurança na universidade & & & & $0,000^{*}$ \\
\hline Sim & $75(47,5)$ & 1 & & \\
\hline Não & $83(52,5)$ & 2,891 & $1,958-4,268$ & \\
\hline Tratamento entre os universitários & & & & $0,000^{*}$ \\
\hline Mal & $49(31,0)$ & 1 & & \\
\hline Bem & $109(69,0)$ & 0,275 & $0,172-0,442$ & \\
\hline Tratamento entre universitários e professores & & & & $0,000^{*}$ \\
\hline Mal & $33(20,9)$ & 1 & & \\
\hline Bem & $125(79,1)$ & 0,376 & $0,222-0,638$ & \\
\hline
\end{tabular}

OR: odds ratio; IC95\%: intervalo de confiança de $95 \%$. ${ }^{*} p<0,05$ 
Tabela IV - Análise multivariada da situação de violência na universidade. Fortaleza, Ceará, 2017.

\begin{tabular}{lccc}
\hline \multirow{2}{*}{ Variáveis } & \multicolumn{3}{c}{ Situação de violência na universidade } \\
& OR ajustado & IC95\% & p-valor \\
\hline Semestre & 2,315 & $1,311-4,088$ & $0,004^{*}$ \\
Curso de graduação & 0,967 & $0,834-1,122$ & 0,661 \\
Sensação de segurança na universidade & 1,682 & $0,937-3,017$ & 0,081 \\
Tratamento entre os universitários & 0,476 & $0,228-0,994$ & $0,048^{*}$ \\
Tratamento entre universitários e professores & 0,532 & $0,210-1,349$ & 0,184 \\
\hline
\end{tabular}

OR: odds ratio; IC95\%: intervalo de confiança de $95 \%$. * $p<0,05$

\section{DISCUSSÃO}

A situação de violência é uma pandemia que afeta a todos, independentemente das condições sociais, culturais ou econômicas dos envolvidos, além disso, pode ocorrer em diferentes cenários, dentre eles, o ambiente universitário também é palco de diferentes tipos de violência ${ }^{(7)}$.

No presente estudo, um terço dos estudantes da área da saúde informou existir situação de violência na universidade, destacadamente perpetrada pelos próprios alunos. Esses achados mostraram-se inferiores aos encontrados em estudo com universitários na cidade de São Paulo, Brasil, no qual mais da metade das estudantes já tinha sofrido algum tipo de violência e um terço dos homens já tinham perpetrado algum tipo ${ }^{(9)}$.

Fatores sociodemográficos coletados, como faixa etária, sexo, religião e raça não tiveram relação com a violência autorreferida pelos alunos. No entanto, estudo com universitários paulistas mostrou que condições como morar com os pais, religião e comportamento ético tiveram um efeito protetor em sofrer ou perpetrar atos violentos ${ }^{(9)}$.

Sobre a tipologia da situação de violência, o presente estudo verificou que a psicológica foi a mais relatada. No entanto, pesquisa realizada com estudantes americanos em 17 faculdades observou maior índice de violência sexual e uso de drogas ilícitas, além disso, muitos que praticaram esse tipo de violência já haviam sido vítimas em algum período da vida ${ }^{(10)}$. A violência sexual associada à emocional e física foi verificada também em estudantes de seis universidades espanholas, observando ainda maiores chances de tentativas de suicídio ${ }^{(11)}$.

Grupos vulneráveis foram também investigados no presente estudo, no qual constatou que $30 \%$ a $20 \%$ dos estudantes disseram que os obesos, os homoafetivos e os deficientes físicos seriam alvo de tratamento inadequado.

Sobre esses achados, pesquisa com universitários, docentes e gestão do curso de Odontologia, no Rio de Janeiro, Brasil, de uma instituição pública detectou a existência de violência física entre aluno e professor, situações desrespeitosas no trote, atitudes machistas contra as mulheres e de opressão dirigidas a homossexuais ${ }^{(12)}$. Tais condições também foram verificadas em diversos outros estudos em diferentes cidades do Brasil e do mundo ${ }^{(13-15)}$.

Esses comportamentos inadequados nas relações interpessoais, o bullying, a violência verbal e física durante a formação acadêmica pode repercutir negativamente na qualidade de vida dos estudantes, afetando a saúde mental dessa população e favorecendo o aparecimento de transtornos de natureza psicológica, como a depressão e a síndrome de Burnout ${ }^{(14,16,17)}$.

Os últimos semestres da graduação e o curso apresentaram relação com uma maior proporção de relatos sobre situação de violência na universidade, principalmente nos cursos de Fisioterapia, Medicina e Psicologia. Apesar de expressiva produção científica e indicadores oficiais sobre os atos de violência entre jovens adultos, há escassez de estudos com estudantes de diferentes cursos da área da saúde e em diferentes semestres que pudessem elucidar esses achados.

Estudantes da área de saúde pernambucanos foram investigados sobre violência na universidade e condutas de saúde em relação ao início e ao final do curso, não sendo encontrado diferença entre ingressantes e concludentes. Assim, para esse estudo, os conhecimentos adquiridos durante a graduação não favoreceram condutas saudáveis ${ }^{(18)}$.

Estudo realizado com estudantes de Enfermagem, Medicina e Odontologia, em Santa Catarina, identificou que a maioria já vivenciou situações de violência. Além disso, o estudo detectou que os estudantes desconhecem as diferentes tipologias de violência. Raiva e indignação foram os principais sentimentos, e poucas formas de enfretamento foram percebidas. Esse fenômeno pode interferir na conduta profissional futura desses estudantes, na abordagem e na condução dos pacientes vitimizados. Assim, a discussão sobre violência no cenário universitário é dificultada pela formação no modelo biomédico e inadequação dos conteúdos dos currículos das universidades brasileiras para o exercício de atividades que envolvam a pluralidade das necessidades do indivíduo, inclusive na temática da violência ${ }^{(19)}$. 
No presente estudo verificou-se também o bom tratamento entre os universitários e entre os professores, referido por mais de $80 \%$ da amostra. Além disso, a sensação de segurança na universidade foi apontada por mais de $60 \%$ dos entrevistados.

O bom relacionamento com colegas do curso e com os professores é considerado um preditor de qualidade de vida e de satisfação acadêmica com o curso superior. Ao contrário, um relacionamento abusivo pode gerar solidão, violência e suicídio ${ }^{(20,21)}$. Nessa perspectiva, a universidade se coloca como cenário favorável para abertura de diálogos entre todos os imbricados direta ou indiretamente aos processos educacionais, atuando conjuntamente no enfrentamento, com diálogo e fomento ao amplo discurso de paz ${ }^{(4)}$.

Em face aos contextos pessoais e educacionais, os universitários podem gerenciar conflitos pessoais e internos por meio de técnicas de incorporação de princípios que visam a ajustes comportamentais, integração e satisfação entre colegas, que refletem um desempenho acadêmico satisfatório, enfatizando os relacionamentos interpessoais, tanto com colegas, professores e funcionários, quanto a adaptação e empatia com o ensino superior, contribuindo no desempenho acadêmico e qualidade de aprendizagem ${ }^{(20)}$.

Assim, é fundamental a promoção de estratégias voltadas à melhoria de aspectos que envolvam toda a dimensão de satisfação da instituição, em especial a segurança, conforto, visando melhorar a qualidade do ensino e realmente satisfazer os desejos dos acadêmicos, em associação à responsabilidade da gestão universitária e não uma responsabilidade que deve ser atribuída à coordenação dos cursos ou ao líder dos setores envolvidos ${ }^{(22)}$.

Por fim, a prevenção de violência é fomentada pela colaboração entre as comunidades acadêmicas e práticas entre universitários e profissionais. As parcerias acadêmico-comunitárias também contribuem beneficamente na atuação conjunta destas situações ${ }^{(23)}$.

A limitação desse estudo em questão se refere ao instrumento utilizado para a coleta dos dados, devido a ausência de variáveis que poderiam explicar a visão dos estudantes sobre as situações de violência nesse ambiente e dos atores envolvidos.

\section{CONCLUSÃO}

A situação de violência do tipo psicológica está presente no ambiente universitário estudado, é perpetrada pelos próprios estudantes, estando associada ao último ano do curso de graduação na área da saúde e ao tratamento entre os estudantes.

\section{CONFLITOS DE INTERESSE}

Não há conflitos de interesse.

\section{REFERÊNCIAS}

1. Rosário AC, Candeias A, Melo M. Violência entre pares na adolescência: Um estudo com estudantes no início e no final do $3 .{ }^{\circ}$ ciclo do ensino básico. Psicologia. 2017;31(2):57-67.

2. Silva PLN, Almeida SG, Martins AG, Gamba MA, Alves ECS, Silva Junior RF. Práticas educativas sobre violência contra a mulher na formação de universitários. Rev Bioét. 2016;24(2):276-85.

3. Lima MCP, Ramos-Cerqueira ATA, Dantas CL, Lamardo JR, Reis LEC, Torres AR. O Trote e a Saúde Mental de Estudantes de Medicina. Rev Bras Educ Med. 2017;41(2):210-220.

4. Villaça, FM, Palácios, M. Concepções sobre assédio moral: bullying e trote em uma escola médica. Rev Bras Educ Med. 2016;34(4):506-514.

5. Bandeira LM. Trotes, assédios e violência sexual nos campi universitários no Brasil. Gênero. 2017;17(2):49-79.

6. Albuquerque PP, Williams LC. A. Homofobia na escola: relatos de universitários sobre as piores experiências. Temas Psicol. 2015;23(3):663-676.

7. Scherer ZAP. Manifestação de violência no ambiente universitário: o olhar de acadêmicos de enfermagem. Rev Eletrônica Enferm. 2015;17(1):69-77.

8. Catrib AMF, Olegario NBC, Mont'Alverne DGB, Silva GPF, Abdon APV, Silva JG et al. Desenvolvimento e 
reprodutibilidade do instrumento de avaliação da promoção da saúde na universidade - IAPSU. Rev Bras Promoç Saúde. 2015;28(3):305-317.

9. Zotareli V, Faúndes A, Osis MJD, Duarte GA, Sousa MH. Gender and sexual violence among students at a brazilian university. Rev Bras Saude Mater Infant. 2012;12(1):37-46.

10. Porta CM, Mathiason MA, Lust K, Eisenberg ME. Sexual Violence Among College Students: An Examination of Individual and Institutional Level Factors Associated With Perpetration. Journal of Forensic Nursing. 2017; 13(3): 109-117.

11. Leone JM, Carroll JM. Victimization and suicidality among female college students. J Am Col Health. 2016;64(2):421428.

12. Teixeira MCB, Dias MC, Ribeiro CDM. Entre espelhos: a formação em saúde e sua produção de violência. Revista da Abeno. 2018;18(2):156-165.

13. Costa SM, Dias OV, Dias ACA, Souza, TR, Freitas, DM, Rosa TTAS et al. Identificação de maus-tratos entre acadêmicos de saúde. Rev Fac Ciênc Méd Sorocaba. 2017;19(3):133-8.

14. Mateus GAP, Pingoello I. Ocorrência de bullying no ensino superior. Uningá Review. 2015;22(3):30-36.

15. Nama N, MacPherson P, Sampson M, McMilan HJ. Medical students' perception of lesbian, gay, bisexual, and transgender (LGBT) discrimination in their learning environment and their self-reported comfort level for caring for LGBT patients: a survey study. Med Educ Online. 2017; 22(1):1368850.

16. Peres MFT, Barreto, ADLB, Babler F, Quaresma IYV, Arakaki JNL, Eluf-Neto J. Exposição à violência, qualidade de vida, depressão, e burnout entre estudantes de medicina em uma universidade estadual paulista. Rev Med (São Paulo). 2014;93(3):115-24.

17. Lian Q, Su Q, Li R, Elgar FJ, Liu Z, Zheng D. The association between chronic bullying victimization with weight status and body self-image: a cross-national study in 39 countries. PeerJ. 2018;6:e4330.

18. Franca $C$, Colares V. Estudo comparativo de condutas de saúde entre universitários no início e no final do curso. Rev Saúde Pública 2008;42(3)420-7.

19. Rosa R, Boing AF, Schaiber LB, Coelho EBS. Violência: conceito e vivência entre acadêmicos da área da saúde. Interface - Comunic Saude Educ. 2010;14 (32)81-90.

20. Bardagi MP, Hutz CS. Rotina acadêmica e relação com colegas e professores Impacto na evasão universitária. Psico. 2012;43(2):174-184.

21. Guimarães SER, Boruchovitch E. O estilo motivacional do professor e a motivação intrínseca dos estudantes: uma perspectiva da teoria da autodeterminação. Psicol Reflex Crit. 2004;17(2):143-150.

22. Fadel CB, Souza JA, Bordin D, Garbin CAS, Garbin AJI, Saliba NA. Satisfaction with the academic experience among graduate students of a brazilian public university. RGO, Rev. Gaúch Odontol. 2018;66(1):50-59.

23. Smith LS, Wilkins N, Marshall SW, Dellapenna A, Pressley JC, Bauer M, et al. The power of academic-practitioner collaboration to enhance science and practice integration: injury and violence prevention case studies. J Public Health Manag Pract. 2018;24(Suppl):S67-S74.

\section{Endereço para correspondência:}

Carla Christina Pereira da Silva Godinho

Universidade de Fortaleza - UNIFOR

Programa de Pós-Graduação em Saúde Coletiva

Av. Washington Soares, 1321, Bloco S, Sala 1

Bairro: Edson Queiroz

CEP: 60.811-905 - Fortaleza - CE - Brasil

E-mail: soraya_trajano@hotmail.com 